\title{
Erratum to: Multivariate Extremes of Random Scores of Particles in Branching Processes with Max-Linear Heredity
}

\author{
A. V. Lebedev ${ }^{1^{*}}$ \\ ${ }^{1}$ Lomonosov Moscow State University, Moscow, 119991 Russia \\ Received November 4, 2019; in final form, November 4, 2019; accepted January 21, 2020
}

DOI: $10.1134 / \mathrm{S} 0001434620050399$

The original article can be found online at https://doi.org/10.1134/S0001434619030088

In my paper, it is necessary to make the following corrections on p. 382. In Example 3 (line 6 from below), the equality $b_{k}=\mu^{-k}$ must be replaced by $b_{k}=\mu^{-k} \mathrm{P}\left(Z_{k}>0\right)$; therefore, instead of the formula (line 3 from below),

$$
\theta=\sum_{k=0}^{\infty} a_{k}^{\gamma} \mu^{-k}
$$

we have

$$
\theta=\sum_{k=0}^{\infty} a_{k}^{\gamma} \mu^{-k} \mathrm{P}\left(Z_{k}>0\right) .
$$

These formulas yield the same results only for immortal branching processes (when each particle has at least one descendent), and the formula

$$
\theta=\frac{1-a^{\gamma}}{1-a^{\gamma} / \mu}
$$

in the max-autoregressive model (line 1 from below) is also valid for immortal branching processes.

\footnotetext{
*E-mail: avlebed@yandex.ru
} 